\title{
The YKL-40 protein is a potential biomarker for COPD: a meta-analysis and systematic review
}

This article was published in the following Dove Press journal:

International Journal of COPD

\author{
Xiang Tong' \\ Dongguang Wang' \\ Sitong Liu' \\ Yao $\mathrm{Ma}^{1,2}$ \\ Zhenzhen $\mathrm{Li}^{3}$ \\ Panwen Tian ${ }^{1,4}$ \\ Hong Fan' \\ 'Department of Respiratory and \\ Critical Care Medicine, West \\ China Hospital/West China School \\ of Medicine, Sichuan University, \\ Chengdu, People's Republic of China; \\ ${ }^{2}$ The Center of Gerontology and \\ Geriatrics, West China Hospital/West \\ China School of Medicine, Sichuan \\ University, Chengdu, People's Republic \\ of China; ${ }^{3}$ Health Management \\ Center, West China Hospital/West \\ China School of Medicine, Sichuan \\ University, Chengdu, People's Republic \\ of China; ${ }^{4}$ Lung Cancer Center, West \\ China Hospital/West China School \\ of Medicine, Sichuan University, \\ Chengdu, People's Republic of China
}

Background: Many studies have found that YKL-40 may play an important pathogenic role in COPD. However, the results of these studies were inconsistent. Therefore, we performed a systematic review and meta-analysis to investigate the role of YKL-40 in COPD.

Methods: We performed a systematic literature search in many database and commercial internet search engines to identify studies involving the role of YKL-40 in patients with COPD. The standardized mean difference (SMD) and Fisher's Z-value with its 95\% confidence interval (CI) were used to investigate the effect sizes.

Results: A total of 15 eligible articles including 16 case-control/cohort groups were included in the meta-analysis. The results indicated that the serum YKL-40 levels in patients with COPD were significantly higher than those in healthy controls (SMD $=1.58,95 \% \mathrm{CI}=0.68-2.49$, $P=0.001$ ), and it was correlated with lung function (pooled $r=-0.32 ; Z=-0.33 ; P<0.001$ ). The results of subgroup analysis found that the serum YKL-40 levels were statistically different between the exacerbation group and the stable group in patients with COPD (SMD $=1.55$, $95 \% \mathrm{CI}=0.81-2.30, P<0.001)$. Moreover, the results indicated that the sputum YKL-40 levels in patients with COPD were also significantly higher than those in healthy controls ( $\mathrm{SMD}=0.70$, 95\% CI $=0.10-1.30, P=0.022$ ).

Conclusion: The current study suggests that YKL-40 may be implicated in bronchial inflammation and remodeling in COPD and may be considered as a useful biomarker for COPD diagnosis and monitoring.

Keywords: YKL-40, COPD, biomarker, meta-analysis

\section{Introduction}

COPD is one of the leading causes of chronic respiratory health issues worldwide, with an increasingly high prevalence. ${ }^{1}$ It is characterized physiologically by chronic airway inflammation involving the central and the peripheral airways that lead to largely irreversible and progressive airflow limitation. ${ }^{2}$ Additionally, several airway structural changes, known as remodeling, can be observed in COPD. ${ }^{2}$ In fact, despite continuing advances in medicine, many patients with COPD experience high levels of mortality, comorbidities, and a substantial health-economic burden., ${ }^{1,3}$ Patient prognosis could be improved if diagnosed at the earliest stage or well monitored. Therefore, there is intense interest in exploring specific and novel biomarkers attributed to COPD, for early diagnosis, optimizing and monitoring its medical management and outcomes. A series of studies has indicated many biomarkers considered as prognosticators, as well as indicators of screening and potential therapeutic targets for COPD, such as C-reactive protein, vitamin D, and sputum neutrophils. ${ }^{4-6}$ In recent years, YKL-40, also referred to as chitinase-3-like-1 protein (CHI3L1) and human cartilage glycoprotein-39 (HCgp-39), has attracted attention as a useful biomarker of diseases. 
YKL-40 is a member of the mammalian chitinase-like protein family, and its encoding gene is located on chromosome 1q32.1. ${ }^{7}$ It is produced by various cell types, including macrophages, neutrophils, monocytes, airway epithelium, vascular smooth muscle cells, synovial cells, chondrocytes, and breast cells. ${ }^{8}{ }^{89}$ The exact function of YKL-40 is not completely clear, but as an inflammatory glycoprotein, it is thought to be involved in many pathophysiological processes, including cell growth, migration, chemotaxis, reorganization, and tissue remodeling. ${ }^{10,11}$ Previous studies have demonstrated that there are elevated blood YKL-40 levels in various diseases, which are characterized by inflammation, abnormal cell growth, and tissue remodeling, such as cardiovascular disease, diabetes, cancer, and idiopathic pulmonary fibrosis. ${ }^{12-14}$ Additionally, a growing body of research has found that YKL-40 may play an important pathogenic role in the establishment of bronchial inflammation and remodeling in patients with COPD.${ }^{15}$ However, most of these studies have been small in sample size, and a single study may lack sufficient statistical power to detect the potentially small effect of the YKL-40 levels on COPD. In addition, the results of these studies were inconsistent. Therefore, to overcome these and other limitations and to better understand the role of YKL-40 in COPD, we conducted a systematic review and meta-analysis to accurately investigate the association between YKL-40 and patients with COPD.

\section{Methods}

\section{Literature search}

We performed a systematic literature search in the PubMed, Embase, Web of Science, Ovid Medline, Wanfang database (www.wanfangdata.com.cn), and the China National Knowledge Infrastructure (CNKI, www.cnki.net) to identify studies involving the role of YKL-40 in patients with COPD, with the most recent search having been conducted on April 21, 2017. The key search terms were as follows: (chronic obstructive pulmonary disease OR COPD OR chronic airway inflammatory disease) AND (YKL-40 OR CHI3L1 OR HCgp-39 OR chitinase-3-like-1 protein OR human cartilage glycoprotein-39). The language was restricted to English or Chinese. Moreover, we conducted a web-based search in several commercial Internet search engines (such as Baidu Scholar and Google Scholar) using the same keywords. All analyses in the current meta-analysis were based on previously published studies; thus, no ethical approval and patient consent are required.

\section{Study selection}

The inclusion criteria were defined as follows: 1) a study involving the role of YKL-40 in COPD designed as a cohort study or case-control study; 2) if there was duplication of data, only the most complete and recent study was included; and 3) a primary study provided available data for counting standardized mean difference (SMD) with a $95 \%$ confidence interval (CI). The exclusion criteria were as follows: 1) the study was not designed as a cohort study or case-control study; 2) a study did not provide available data for counting effect size or was missing other essential information; and 3) review, abstract, or overlapping study.

\section{Study quality score evaluation}

The qualities of identified studies involving the role of YKL-40 in COPD were evaluated using the NewcastleOttawa Scale (NOS), and the scale to investigate quality was based on three aspects: selection, comparability, and exposure in the primary study. The total score ranged from 0 to 9 (0-3, 4-6, and 7-9 was considered low-, moderate-, and high-quality, respectively). The quality of the studies was assessed in a consensus meeting with all authors.

\section{Data extraction}

Two independent authors (Xiang Tong and Dongguang Wang) extracted the detailed information and data from each primary study using a predesigned data extraction Excel form. If there was any disagreement or doubt, the third author (Sitong Liu) further reviewed these articles. The information and data extraction included the following: first author, year of publication, ethnicity, age of participant, serum, and sputum YKL-40 levels, correlation coefficient (r), sample size of patients and controls, and Fisher's $Z$-value, and method of YKL-40 measurement.

If a study only provided medians and ranges (or interquartile ranges [IQR]), we transformed the data to approximate means and standard deviations following the validated methods proposed by Wan et al. ${ }^{16}$ Additionally, if the Fisher's $Z$-value could not be directly obtained from the publication, Fisher's $r$-to- $Z$ transformation was used to convert each correlation coefficient into their approximately associated $Z$ statistics, which is considered the normal distribution. ${ }^{17}$

\section{Statistical methods}

In the present study, all data analyses were performed using STATA 12.0 and SPSS 21.0 software. After appropriate conversion, we chose the random-effect model to synthesize 
data from the various studies because it is thought to be more conservative, incorporates better between-study variance terms, and produces a lower type I error rate as well as a wider CI for the pooled effects estimate when compared with the fixed-effect model. ${ }^{18}$ The SMD with $95 \%$ CI was used to compare the levels of serum or sputum YKL-40 in the COPD patients with the levels in healthy population. Regarding the pooled correlation coefficient, we used Fisher's $Z$-value to calculate the related statistic (summary $Z$-value) and $95 \% \mathrm{CI}$ by the methods described in the previous study, and then transferred them back to summary effect size (summary $r$-value) using Fisher's $Z$-to- $r$ transformation procedure. ${ }^{17}$

The between-study heterogeneity was investigated by the chi-squared-based $Q$-test and $I$-squared $\left(I^{2}\right)$ statistics test. A $P$-value of $<0.10$ or an $I^{2}$-value of $>50 \%$ suggested a statistically significant heterogeneity. If a notable heterogeneity was found, we used a Kruskal-Wallis test to directly compare the levels of serum YKL-40 in the COPD patients with the levels in controls and reported the results of this analysis as the median and IQR. Additionally, meta-regression analysis and sensitivity analysis were used to investigate the sources of heterogeneity. We further carried out subgroup analysis to determine the effect of status of disease (exacerbation vs stable). Moreover, the visual inspection of asymmetry in funnel plots was used to assess publication bias, and the Begg's and Egger's tests were used to further detect publication bias.

\section{Results \\ Study characteristics}

A total of 119 articles were identified in the initially searches in PubMed, Embase, Web of Science, Ovid Medline, Wanfang database, CNKI, and commercial Internet search engines. As shown in Figure 1, 80 studies were excluded because they were duplicated across the databases, and 4 publications were excluded because they were various conference abstracts. After screening on title and abstract, 7 articles were excluded because they were not relevant to serum/sputum YKL-40 in relation to COPD. The remaining 28 articles were further screened by a full view. Nine articles were removed because they were editorials and reviews. Two articles were excluded because they only reported the relationship between $Y K L-40$ gene polymorphism and susceptibility to COPD. One study was excluded because the participants of the study were healthy individuals. One article was excluded because there was a potential repetition of data in another study. Therefore, 15 eligible articles ${ }^{15,19-32}$ (13 case-control/cohort groups for serum YKL-40 levels and COPD; 3 case-control/cohort groups for sputum YKL-40

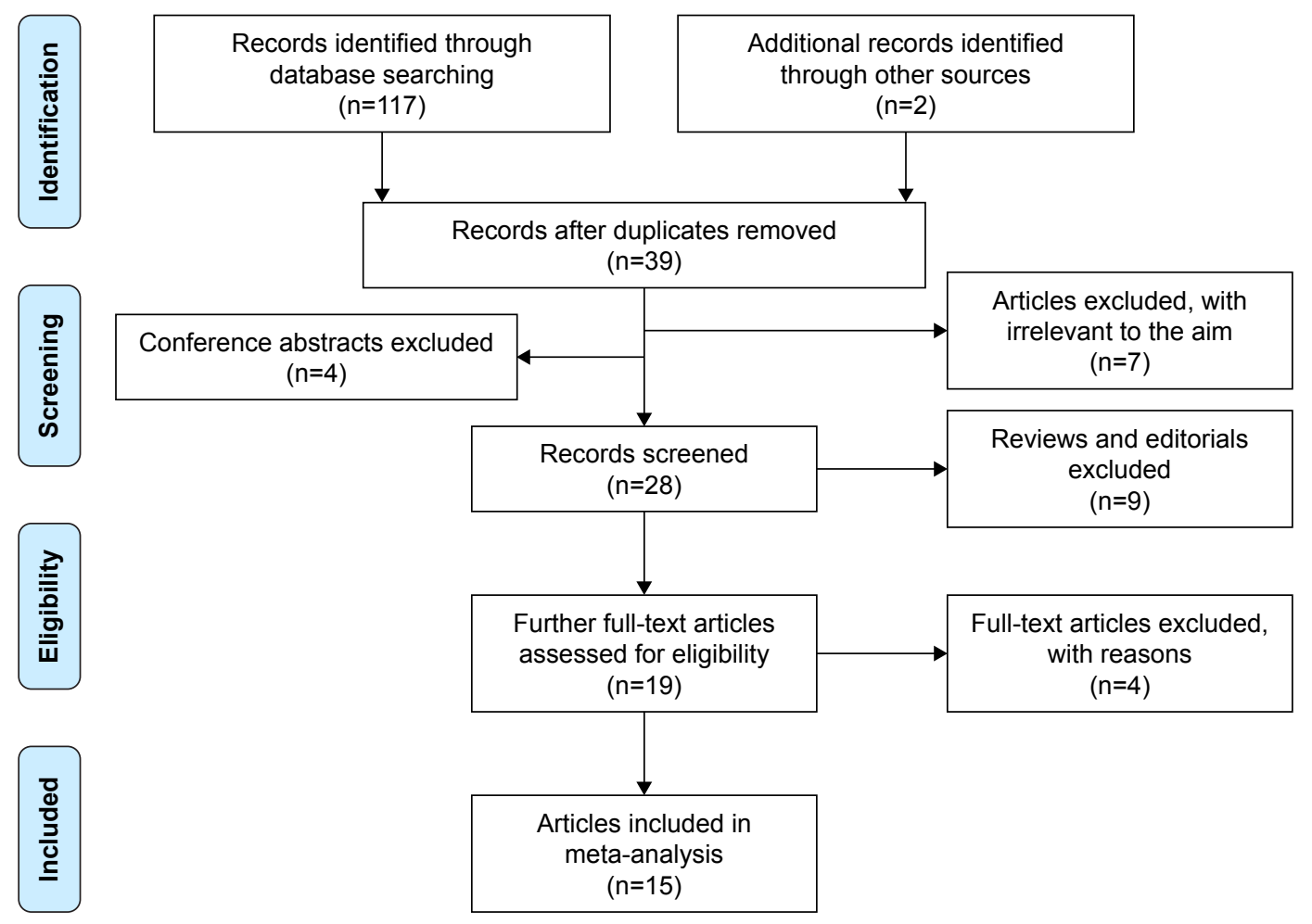

Figure I The flow diagram of included and excluded studies. 
levels and COPD) were finally identified in the current metaanalysis. Five articles (33.3\%) were of moderate quality, and the other included studies (66.7\%) were of high quality according to the NOS quality score evaluation.

\section{Association between serum YKL-40 levels and patients with COPD}

All 13 case-control/cohort studies, including 1,205 cases and 3,600 controls, showed an association between the serum YKL-40 levels and patients with COPD (Table 1). Overall, the meta-analysis results indicated that the serum YKL-40 concentrations in patients with COPD were significantly higher than those in healthy population $(\mathrm{SMD}=1.58$, 95\% CI $=0.68-2.49, P=0.001$ ) (Figure 2). Begg's test found no publication bias $(P=0.721)$, while a publication bias was determined in the results of Egger's tests $(P=0.003)$. A nonignorable heterogeneity between studies was observed $\left(I^{2}=98.5 \%\right)$. Therefore, we compared the levels of serum YKL-40 in the COPD patients with the levels in controls using the Kruskal-Wallis test. The result (COPD group: median $=124.7 \mathrm{ng} / \mathrm{mL}[\mathrm{IQR}=59.3-273.5]$ vs control group: median $=49.9 \mathrm{ng} / \mathrm{mL}[\mathrm{IQR}=26.2-144.7], P=0.018)$ was consistent with that of the current meta-analysis. We further performed a meta-regression analysis and sensitivity analysis to reveal the possible sources of heterogeneity.

\section{Sensitivity and meta-regression analysis}

We carried out a multivariate meta-regression analysis to investigate the possible confounding factors contributing to the heterogeneity. The results demonstrated that the sample size of the ratio of case and control sample sizes, the case group, the year of publication, and study quality as confounding factors did not substantially affect heterogeneity (adjusted $P$-values were $0.983,0.904,0.948$, and 0.996 for COPD). Moreover, we performed a sensitivity analysis by sequentially extracting each study from the meta-analysis to explore the effect of every study on the pooled meta-results. The sensitivity analysis suggested that the pooled SMD were not materially altered, indicating the stability of our meta-analysis (Figure 3).

\section{Subgroup meta-analysis}

Because of the notable heterogeneity, we further performed subgroup analysis that was expected to lead to heterogeneity. In the subgroup analysis of disease status (exacerbation vs stable) (Table 2), the results showed that the levels of serum YKL-40 were statistically different between the exacerbation group and the stable group in patients with COPD ( $\mathrm{SMD}=1.55,95 \% \mathrm{CI}=0.81-2.30, P<0.001)$ (Figure 4).

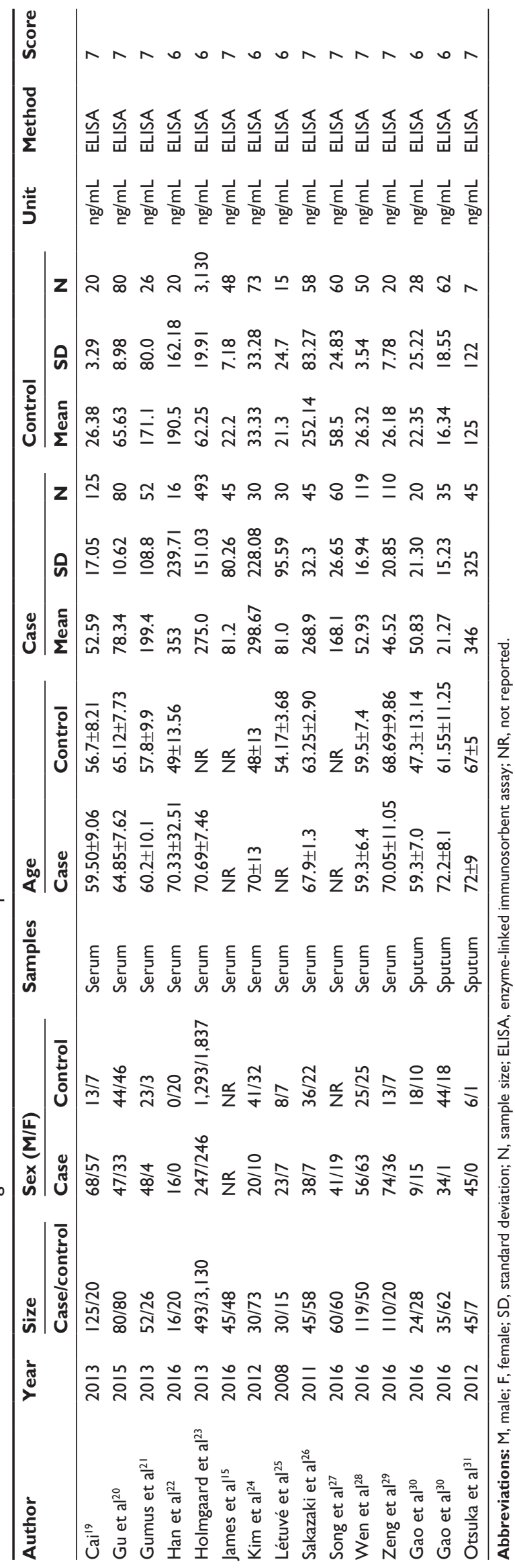




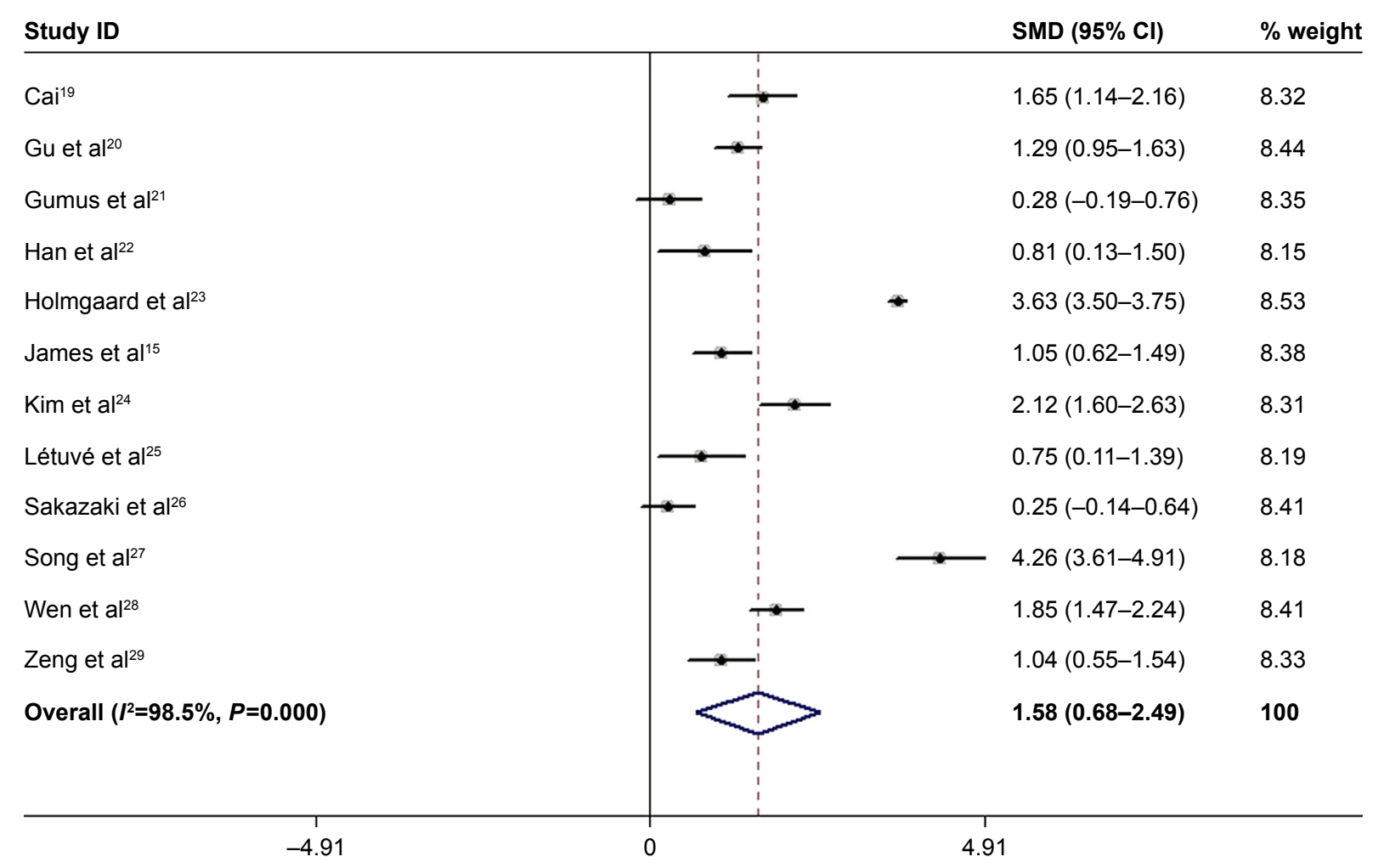

Figure 2 The results of association between serum YKL-40 levels and COPD.

Note: Weights are from random effects analysis.

Abbreviations: SMD, standardized mean difference; $\mathrm{Cl}$, confidence interval.

\section{Meta-analysis estimates, given named study is omitted}

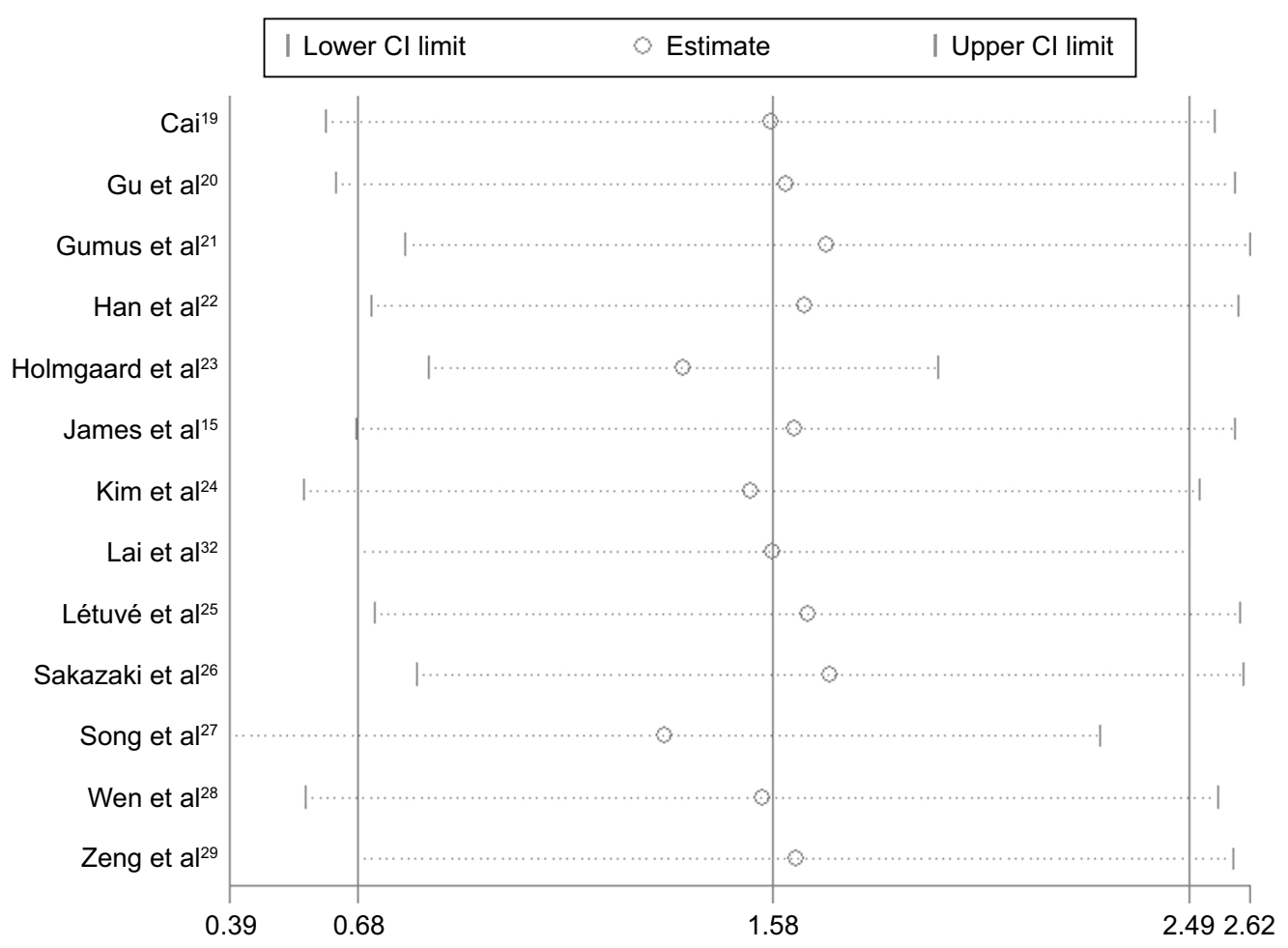

Figure 3 The result of sensitivity analysis on association between serum YKL-40 levels and COPD. Abbreviation: $\mathrm{Cl}$, confidence interval. 
Table 2 Characteristics of studies involving in comparison of the differences of serum YKL-40 levels in the exacerbation group and the stable group

\begin{tabular}{|c|c|c|c|c|c|c|c|c|c|c|}
\hline \multirow[t]{2}{*}{ Author } & \multirow[t]{2}{*}{ Year } & \multirow{2}{*}{$\frac{\text { Size }}{\text { Case/control }}$} & \multicolumn{2}{|c|}{$\operatorname{Sex}(M / F)$} & \multicolumn{3}{|l|}{ Stable } & \multicolumn{3}{|c|}{ Exacerbation } \\
\hline & & & Case & Control & $\begin{array}{l}\text { Mean } \\
\text { (ng/mL) }\end{array}$ & SD & $\mathbf{N}$ & $\begin{array}{l}\text { Mean } \\
(\mathrm{ng} / \mathrm{mL})\end{array}$ & SD & $\mathbf{N}$ \\
\hline $\mathrm{Cai}^{19}$ & 2013 & $125 / 20$ & $68 / 57$ & $13 / 7$ & 40.40 & 8.83 & 65 & 65.8 & 13.60 & 60 \\
\hline Lai et $\mathrm{al}^{32}$ & 2016 & $81 / 47$ & $54 / 27$ & $28 / 19$ & 51.13 & 33.95 & 44 & 84.37 & 53.91 & 37 \\
\hline Wen et $\mathrm{al}^{28}$ & 2016 & $119 / 50$ & $56 / 63$ & $25 / 25$ & 40.32 & 8.43 & 59 & 65.32 & 13.74 & 60 \\
\hline Zeng et $\mathrm{al}^{29}$ & 2016 & $110 / 20$ & $74 / 36$ & $13 / 7$ & 36 & 16.64 & 50 & 55.29 & 20.02 & 60 \\
\hline
\end{tabular}

Abbreviations: $M$, male; $F$, female; SD, standard deviation; $N$, sample size.

\section{Meta-analysis of correlations between serum YKL-40 levels and clinical parameters}

Regarding the correlations between serum YKL-40 levels and clinical parameters in patients with COPD, the results suggested that elevated levels of serum YKL-40 were

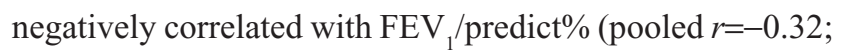
$Z=-0.33 ; P<0.001$ ) (Figure 5), while they were not correlated with $\mathrm{FEV}_{1}$ (pooled $r=-0.15 ; Z=-0.15 ; P=0.238$ ), blood IgE concentrations (pooled $r=0.29 ; Z=0.30 ; P=0.019$ ), or blood eosinophil counts (pooled $r=-0.10 ; Z=-0.10 ; P=0.435$ ). However, it should be noted that there was only one included study that included these results $\left(\mathrm{FEV}_{1}\right.$, blood IgE, levels and blood eosinophil counts).

\section{Association between sputum YKL-40 levels and patients with COPD}

A total of 3 case-control/cohort studies, including 104 cases and 97 controls, presented an association between the sputum YKL-40 concentrations and patients with COPD. The meta-analysis results indicated that the levels of sputum YKL-40 in patients with COPD were significantly higher than those in healthy controls (SMD $=0.70,95 \% \mathrm{CI}=0.10-1.30$, $P=0.022$ ) (Figure 6). No heterogeneity was observed between studies.

\section{Discussion}

The present study was conducted to investigate the potential role of YKL-40 in COPD patients. The main findings of this meta-analysis were as follows: 1) the patients with COPD had higher serum YKL-40 concentrations than those in controls; 2) the serum YKL-40 levels were increased in the exacerbation group when compared with the stable group; 3) the elevated serum YKL-40 concentrations were correlated with reduced lung function; and 4) the levels of sputum YKL-40 in patients with COPD were higher than those in the healthy controls.

YKL-40, a chitinase-like protein that was first discovered in mouse breast cancer cells, has the ability to bind chitin,

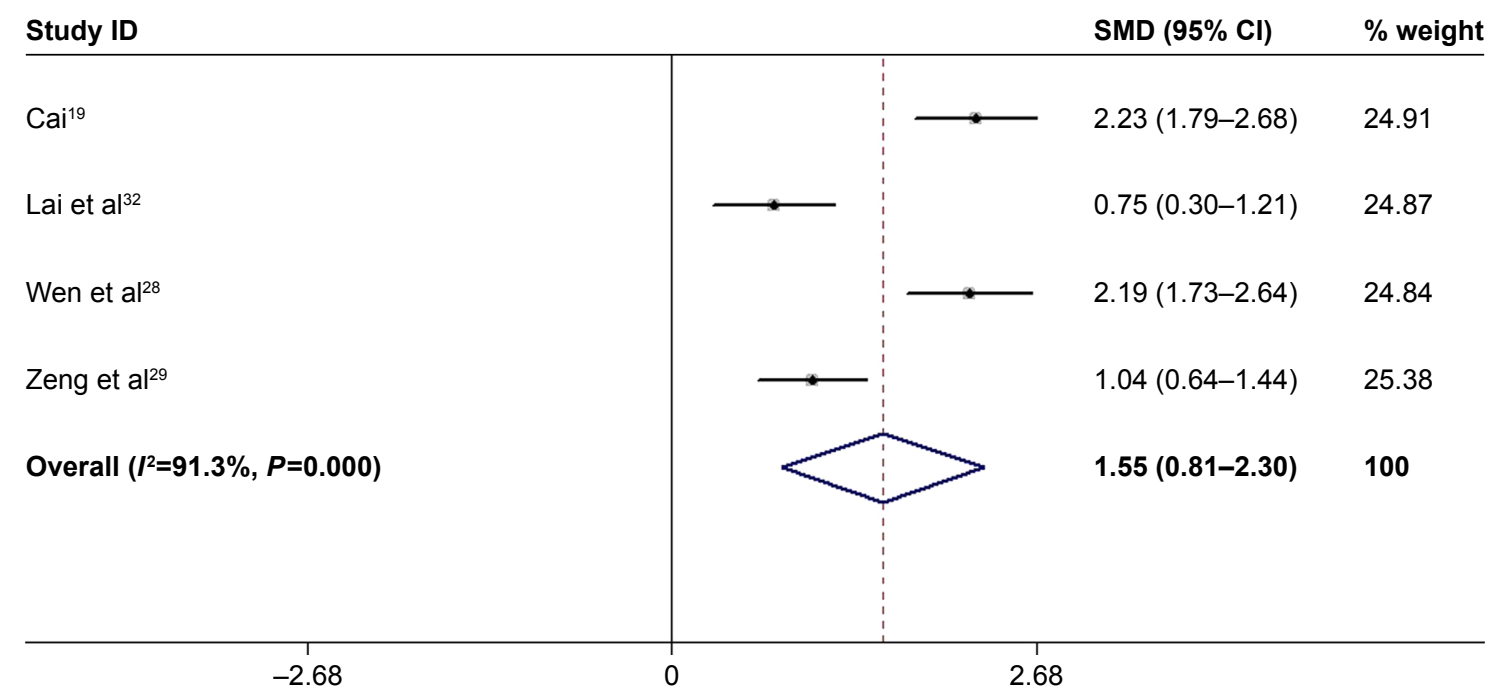

Figure 4 Comparison of the differences of serum YKL-40 levels in the exacerbation group and the stable group. Note: Weights are from random effects analysis.

Abbreviations: SMD, standardized mean difference; $\mathrm{Cl}$, confidence interval. 


\begin{tabular}{|c|c|c|}
\hline Study ID & $\begin{array}{l}\text { Summary } \\
\text { Fisher Z }(95 \% \mathrm{Cl})\end{array}$ & $\%$ weight \\
\hline Gu et $\mathrm{al}^{20}$ & $-0.31(-0.53$ to -0.08$)$ & 25.03 \\
\hline Gumus et $\mathrm{al}^{21}$ & $-0.28(-0.56$ to -0.00$)$ & 15.91 \\
\hline James et $\mathrm{al}^{15}$ & $-0.19(-0.44-0.06)$ & 19.86 \\
\hline Lai et $\mathrm{al}^{32}$ & $-0.47(-0.70$ to -0.25$)$ & 25.48 \\
\hline Sakazaki et al ${ }^{26}$ & $-0.35(-0.65$ to -0.05$)$ & 13.72 \\
\hline Overall $\left(I^{2}=0.0 \%, P=0.562\right)$ & $-0.33(-0.44$ to -0.22$)$ & 100 \\
\hline
\end{tabular}

Figure 5 Pooled correlation coefficients between serum YKL-40 levels and FEV,/predict\%. Note: Weights are from random effects analysis.

Abbreviation: $\mathrm{Cl}$, confidence interval.

but it lacks enzymatic activity, which is important in the homeostasis of many organs and systems. ${ }^{33}$ Several studies have indicated that elevated serum YKL-40 levels were observed in many human diseases, such as communityacquired pneumonia and rheumatoid arthritis, ${ }^{34,35}$ as well as in COPD. ${ }^{15}$ Consistent with previous studies, in our current meta-analysis, we further confirmed that both levels of serum and sputum YKL-40 were increased in patients with COPD, and that these elevated levels were significantly correlated with disease status and lung function. Previous in vitro and in vivo studies showed that transforming growth factor- $\beta$, tumor necrosis factor- $\alpha$, interleukin, and other multifunctional cytokines could also stimulate YKL-40 secretion, ${ }^{36,37}$ and YKL-40 further promoted the expression of macrophage inflammatory protein- $1 \alpha$, monocyte chemoattractant protein-1, and metalloproteinase- $9 .{ }^{25,38}$ In addition, YKL-40 secretion may regulate a series of pathways, including activation of the mitogen-activated protein kinase (MAPK), nuclear factor- $\mathrm{KB}(\mathrm{NF}-\mathrm{\kappa B})$, protein kinase $\mathrm{B}(\mathrm{PKB} / \mathrm{Akt})$, and other cytokine pathways. ${ }^{8,38}$ These all above cytokines and signaling pathways have been found to be closely related to the pathogenesis of COPD, which strongly suggest that YKL-40 may be involved in the critical inflammatory pathophysiology of the disease.

On the other hand, airway remodeling was considered to be another characteristic of COPD that led to irreversible

\begin{tabular}{|c|c|c|}
\hline Study ID & SMD $(95 \% \mathrm{Cl})$ & $\%$ weight \\
\hline Gao et $\mathrm{al}^{30}$ & $1.20(0.58-1.83)$ & 32.75 \\
\hline Gao et $\mathrm{al}^{30}$ & $0.28(-0.13-0.70)$ & 40.90 \\
\hline Otsuka et $a^{31}$ & $0.72(-0.09-1.53)$ & 26.34 \\
\hline Overall $\left(I^{2}=66.0 \%, P=0.053\right)$ & $0.70(0.10-1.30)$ & 100 \\
\hline-1.83 & 1.83 & \\
\hline
\end{tabular}

Figure 6 The results of association between sputum YKL-40 levels and COPD.

Note: Weights are from random effects analysis.

Abbreviations: SMD, standardized mean difference; $\mathrm{Cl}$, confidence interval. 
airflow obstruction. Recently, a large number of studies demonstrated that YKL-40 was associated with pathologic tissue remodeling, and it had an important role in COPD pathogenesis. Bara et al found that YKL-40 promoted bronchial smooth muscle (BSM) cell proliferation and migration by a protease-activated receptor type 2-dependent mechanism. ${ }^{39}$ A recent study by Tang et $\mathrm{al}^{41}$ reported that YKL-40 could result in overexpression of interleukin and was involved in the proliferation and migration of BSM cells through the activation of MAPK, and NF- $\kappa B$ pathways. Additionally, Lai et al reported that the expression of YKL-40 may contribute to collagen production of lung fibroblasts in patients with COPD by ERK- and p38-dependent pathways. ${ }^{32}$ Moreover, airway remodeling was associated with poor prognosis in patients with COPD..$^{40}$ In our current meta-analysis, we confirmed that the elevated levels of serum YKL-40 were associated with clinical severity and that they were correlated with reduced lung function.

Interestingly, Létuvé et al suggested that the YKL-40 levels of bronchoalveolar lavage fluid (BALF) were increased in patients with COPD, and the elevated YKL-40 levels were related to disease severity. ${ }^{25}$ However, in a small size study performed by Otsuka et al, the elevated sputum YKL-40 levels were only found to be associated with airflow obstruction in asthma, while no correlations were found between sputum YKL-40 levels, lung function, and clinical severity in COPD patients. ${ }^{31}$ Our current study (only included 3 studies) found that sputum YKL-40 levels were increased in patients with COPD. In fact, it was not clear whether the sputum YKL-40 was correlated with clinical characteristics or serum YKL-40 nowadays. Although these results were still inconsistent, they seem to point out that the levels of YKL-40 in sputum and BALF may also be considered as an independent local biomarker for COPD reflecting airway inflammation and remodeling.

Taking the results of these previous studies and those of our present study together, it is reasonable to believe that the YKL-40 may be involved in the pathogenesis of COPD, and the elevated serum YKL-40 levels may possibly predict the diagnosis and progression of the disease. Therefore, even though the mechanism remains completely unknown, we speculate that YKL-40 could secrete and regulate many cytokines and specific cells (such as smooth muscle cell) by the activation of a range of signaling pathways to promote tissue inflammation and remodeling.

In the present meta-analysis, it should be noted that there was a non-ignorable heterogeneity between included studies. Although the heterogeneity can be taken into account using the random-effect model, this increases the probability of type I error. The significant heterogeneity may be attributed to many factors, including the following: 1) the different demographic characteristics and ethnic backgrounds in included participants; 2) the different disease statuses (exacerbation vs stable) were included in the studies; 3 ) the included patients and controls had different baseline characteristics (such as age, body mass index, ratios of smoking, symptom score, and lung function) in each study; 4) there were different ratios of sex in each primary study; 5) the included patients had different treatment statuses; 6) many studies did not directly provide available data, but through the statistics transformation was carried out following the validated methods; 7) different measuring methods were used in the primary studies, and the value of YKL-40 levels varied in a comparatively large range; and 8) the included studies were of differing quality. We have used several methods, including sensitivity analysis, meta-regression analysis, and subgroup analysis, to investigate the possible sources of heterogeneity, and as far as possible, to reduce the occurrence of type I error in the present study.

There were several limitations of this meta-analysis. First, only published studies were identified in a few databases, and there were small sample sizes in most included studies, which may explain the publication bias in the current article. These limitations might also lead to additional biases or overall overestimated associations. Second, the identified language was restricted to English and Chinese, which may have screened out valuable articles published in other languages, and it also contributed to the publication bias. Third, because primary studies lacked sufficient data, we failed to further perform subgroup analyses to investigate the other factors, such as sex, age, body mass index, and phenotype, which may have affected our results. Despite these limitations, we minimized the likelihood of bias throughout the entire process by establishing a detailed protocol and conducting study screening, data selection, and statistical analysis, and we controlled for publication bias.

\section{Conclusion}

In summary, the present meta-analysis and systematic review suggest that YKL-40 may be involved in the critical pathogenesis of COPD, including bronchial inflammation and remodeling, it may considered as a useful biomarker for COPD diagnosis, and it was also a potential target for treatment. Further rigorous and uniform studies are needed to better explore the association between YKL-40 and COPD and to investigate whether monitoring of its levels could facilitate successful clinical decisions. 


\section{Acknowledgments}

This study was supported by National Key R\&D Program of China (2017YFC1309703) and the Bureau of Human Resources and Social Security of Sichuan Province Grant (2015-100-13). We would like to thank Elsevier Language Editing Services for its linguistic assistance during the preparation of this manuscript.

\section{Disclosure}

The authors report no conflicts of interest in this work.

\section{References}

1. Mannino DM, Buist AS. Global burden of COPD: risk factors, prevalence, and future trends. Lancet. 2007;370(9589):765-773.

2. Zhou-Suckow Z, Duerr J, Hagner M, Agrawal R, Mall MA. Airway mucus, inflammation and remodeling: emerging links in the pathogenesis of chronic lung diseases. Cell Tissue Res. 2017;367(3): 537-550.

3. Ehteshami-Afshar S, FitzGerald JM, Doyle-Waters MM, Sadatsafavi M. The global economic burden of asthma and chronic obstructive pulmonary disease. Int J Tuberc Lung Dis. 2016;20(1):11-23.

4. Peng C, Tian C, Zhang Y, Yang X, Feng Y, Fan H. C-reactive protein levels predict bacterial exacerbation in patients with chronic obstructive pulmonary disease. Am J Med Sci. 2013;345(3):190-194.

5. Verrills NM, Irwin JA, He XY, et al. Identification of novel diagnostic biomarkers for asthma and chronic obstructive pulmonary disease. Am J Respir Crit Care Med. 2011;183(12):1633-1643.

6. Singh D, Edwards L, Tal-Singer R, Rennard S. Sputum neutrophils as a biomarker in COPD: findings from the ECLIPSE study. Respir Res. 2010;11:77.

7. Rehli M, Krause SW, Andreesen R. Molecular characterization of the gene for human cartilage gp-39 (CHI3L1), a member of the chitinase protein family and marker for late stages of macrophage differentiation. Genomics. 1997;43(2):221-225.

8. Recklies $\mathrm{AD}$, White $\mathrm{C}$, Ling $\mathrm{H}$. The chitinase 3-like protein human cartilage glycoprotein 39 (HC-gp39) stimulates proliferation of human connective-tissue cells and activates both extracellular signal-regulated kinase- and protein kinase B-mediated signalling pathways. Biochem J. 2002;365(1):119-126.

9. Shao R, Hamel K, Petersen L, et al. YKL-40, a secreted glycoprotein, promotes tumor angiogenesis. Oncogene. 2009;28(50):4456-4468.

10. Johansen JS, Schultz NA, Jensen BV. Plasma YKL-40: a potential new cancer biomarker? Future Oncol. 2009;5(7):1065-1082.

11. Nishikawa KC, Millis AJ. gp38k (CHI3L1) is a novel adhesion and migration factor for vascular cells. Exp Cell Res. 2003;287(1): 79-87.

12. Catalán V, Gómez-Ambrosi J, Rodríguez A, et al. Increased circulating and visceral adipose tissue expression levels of YKL-40 in obesityassociated type 2 diabetes are related to inflammation: impact of conventional weight loss and gastric bypass. J Clin Endocrinol Metab. 2011; 96(1):200-209.

13. Furuhashi K, Suda T, Nakamura Y, et al. Increased expression of YKL-40, a chitinase-like protein, in serum and lung of patients with idiopathic pulmonary fibrosis. Respir Med. 2010;104(8):1204-1210.

14. Zheng JL, $\mathrm{Lu} \mathrm{L}, \mathrm{Hu}$ J, et al. Increased serum YKL-40 and C-reactive protein levels are associated with angiographic lesion progression in patients with coronary artery disease. Atherosclerosis. 2010;210(2): 590-595.

15. James AJ, Reinius LE, Verhoek M, et al. Increased YKL-40 and chitotriosidase in asthma and chronic obstructive pulmonary disease. $\mathrm{Am}$ J Respir Crit Care Med. 2016;193(2):131-142.
16. Wan X, Wang W, Liu J, Tong T. Estimating the sample mean and standard deviation from the sample size, median, range and/or interquartile range. BMC Med Res Methodol. 2014;14:135.

17. Wikipedia. Fisher transformation. Available from: https://en.wikipedia. org/wiki/Fisher_transformation

18. Borenstein M, Hedges L, Rothstein H. Meta-analysis: fixed effect vs. random effects. Available from: Meta-analysis.com. 2007. Accessed June 17, 2017

19. Cai Y. Serum YKL-40 Levels and its Significance in COPD patients [dissertation]. Sichuan: Luzhou Medical College; 2013. Chinese.

20. Gu HT, Shao HY, Jin XH, Mao WW. The relationship between chitinase and the severity of chronic obstructive pulmonary disease. $J$ Chin Physician. 2015;17(11):1727-1729. Chinese [with English abstract].

21. Gumus A, Kayhan S, Cinarka H, et al. High serum YKL-40 level in patients with COPD is related to hypoxemia and disease severity. Tohoku J Exp Med. 2013;229(2):163-170.

22. Han SS, Lee WH, Hong Y, et al. Comparison of serum biomarkers between patients with asthma and with chronic obstructive pulmonary disease. J Asthma. 2016;53(6):583-588.

23. Holmgaard DB, Mygind LH, Titlestad IL, et al. Plasma YKL-40 and all-cause mortality in patients with chronic obstructive pulmonary disease. BMC Pulm Med. 2013;13:77.

24. Kim HR, Jun CD, Lee KS, et al. Levels of YKL-40 in pleural effusions and blood from patients with pulmonary or pleural disease. Cytokine. 2012;58(3):336-343.

25. Létuvé S, Kozhich A, Arouche N, et al. YKL-40 is elevated in patients with chronic obstructive pulmonary disease and activates alveolar macrophages. J Immunol. 2008;181(7):5167-5173.

26. Sakazaki Y, Hoshino T, Takei S, et al. Overexpression of chitinase 3-like 1/YKL-40 in lung-specific IL-18-transgenic mice, smokers and COPD. PLoS One. 2011;6(9):e24177.

27. Song QJ, Duan ZM, Liu CJ. Serum YKL 40 expression in chronic obstructive pulmonary disease. Med Assemble. 2016;24(10):213-214. Chinese [with English abstract].

28. Wen JR, Li GM, Xie YP, Tang XL, Wu WQ. Study on YKL40 level change at exacerbation and stable stage of COPD patients. China Med Pharm. 2016;6(15):136-139. Chinese [with English abstract].

29. Zeng QZ, Yang RQ, Liu B, Zheng FD, Zhou RQ. The application of YKL-40 and IL-6 in evaluating the prognosis of patients with chronic obstructive pulmonary disease. J Clin Pulm Med. 2016;21(2):227-230. Chinese [with English abstract].

30. Gao J, Iwamoto H, Koskela J, et al. Characterization of sputum biomarkers for asthma-COPD overlap syndrome. Int J Chron Obstruct Pulmon Dis. 2016;11:2457-2465.

31. Otsuka K, Matsumoto H, Niimi A, et al. Sputum YKL-40 levels and pathophysiology of asthma and chronic obstructive pulmonary disease. Respiration. 2012;83(6):507-519.

32. Lai $\mathrm{T}, \mathrm{Wu} \mathrm{D}$, Chen $\mathrm{M}$, et al. YKL-40 expression in chronic obstructive pulmonary disease: relation to acute exacerbations and airway remodeling. Respir Res. 2016;17:31.

33. Morrison BW, Leder P. Neu and ras initiate murine mammary tumors that share genetic markers generally absent in c-myc and int-2-initiated tumors. Oncogene. 1994;9(12):3417-3426.

34. Nordenbaek C, Johansen JS, Junker P, Borregaard N, Sorensen O, Price PA. YKL-40, a matrix protein of specific granules in neutrophils, is elevated in serum of patients with community-acquired pneumonia requiring hospitalization. $J$ Infect Dis. 1999;180(5):1722-1726.

35. Volck B, Johansen JS, Stoltenberg M, et al. Studies on YKL-40 in knee joints of patients with rheumatoid arthritis and osteoarthritis. Involvement of YKL-40 in the joint pathology. Osteoarthr Cartil. 2001;9(3):203-214.

36. Gratchev A, Schmuttermaier C, Mamidi S, Gooi L, Goerdt S, Kzhyshkowska J. Expression of osteoarthritis marker YKL-39 is stimulated by transforming growth factor beta (TGF-beta) and IL-4 in differentiating macrophages. Biomark Insights. 2008;3:39-44.

37. Zhu Z, Zheng T, Homer RJ, et al. Acidic mammalian chitinase in asthmatic Th2 inflammation and IL-13 pathway activation. Science. 2004;304(5677):1678-1682. 
38. Lee CG, Elias JA. Role of breast regression protein-39/YKL-40 in asthma and allergic responses. Allergy Asthma Immunol Res. 2010; 2(1):20-27.

39. Bara I, Ozier A, Girodet PO, et al. Role of YKL-40 in bronchial smooth muscle remodeling in asthma. Am J Respir Crit Care Med. 2012; 185(7):715-722.

40. Pascual RM, Peters SP. Airway remodeling contributes to the progressive loss of lung function in asthma: an overview. J Allergy Clin Immunol. 2005;116(3):477-486.
41. Tang H, Sun Y, Shi Z, et al. YKL-40 induces IL-8 expression from bronchial epithelium via MAPK (JNK and ERK) and NF- $\kappa B$ pathways, causing bronchial smooth muscle proliferation and migration. J Immunol. 2013;190(1):438-446.

\section{Publish your work in this journal}

The International Journal of COPD is an international, peer-reviewed journal of therapeutics and pharmacology focusing on concise rapid reporting of clinical studies and reviews in COPD. Special focus is given to the pathophysiological processes underlying the disease, intervention programs, patient focused education, and self management protocols.

\section{Dovepress}

This journal is indexed on PubMed Central, MedLine and CAS. The manuscript management system is completely online and includes a very quick and fair peer-review system, which is all easy to use. Visit $\mathrm{http}: / / \mathrm{www}$.dovepress.com/testimonials.php to read real quotes from published authors. 